\title{
The evolution of the physical state of the IGM*
}

\author{
T.-S. Kim ${ }^{1}$, S. Cristiani ${ }^{2,3}$, and S. D’Odorico ${ }^{1}$ \\ 1 European Southern Observatory, Karl-Schwarzschild-Strasse 2, 85748 Garching b. München, Germany \\ e-mail: sdodoric@eso.org \\ 2 ST European Coordinating Facility, ESO, Karl-Schwarzschild-Strasse 2, 85748 Garching b. München, \\ Germany \\ e-mail: scristia@eso.org \\ 3 Osservatorio Astronomico di Trieste, via G. B. Tiepolo 11, 34131 Trieste, Italy
}

Received 25 August 2001 / Accepted 14 December 2001

\begin{abstract}
Using a new, increased dataset of 7 QSOs from VLT/UVES observations combined with one QSO from the literature, the minimum Doppler parameters as a function of neutral hydrogen column density $N_{\mathrm{H} \text { I }}$, $b_{\mathrm{c}}\left(N_{\mathrm{H}}\right)$, of the Ly $\alpha$ forest has been derived at three redshifts $\langle z\rangle=2.1,3.3$ and 3.8. In particular, five QSOs at $\langle z\rangle=2.1$ enable us to study the cosmic variance of $b_{\mathrm{c}}\left(N_{\mathrm{H}_{\mathrm{I}}}\right)$ at lower $z$ for the first time. When incompleteness of the number of the observed lines towards lower $N_{\mathrm{H} \text { I }}$ is accounted for, the derived slopes of $b_{\mathrm{c}}\left(N_{\mathrm{H}}\right),(\Gamma-1)$, are consistent with no- $z$ evolution with an indication of lower value at $\langle z\rangle=3.3$, while $b_{\mathrm{c}}\left(N_{\mathrm{H}_{\mathrm{I}}}\right)$ at a fixed column density $N_{\mathrm{H}}=10^{13.6} \mathrm{~cm}^{-2}, b_{\mathrm{c}}(13.6)$, increases as $z$ decreases. Assuming a QSO-dominated UV background, the slope of the equation of state $(\gamma-1)$ shows no $z$-evolution within large uncertainties and the temperature at the mean density, $T_{0}$, decreases as $z$ decreases at three redshift ranges. There is a large fluctuation of $(\Gamma-1)$ and $b_{\mathrm{c}}(13.6)$ even at the similar redshifts, in particular at $\langle z\rangle=3.3$ and 3.8. The lower $(\Gamma-1)$ and higher $b_{\mathrm{c}}(13.6)$ values at $z \sim 3.1$ and 3.6 compared to ones at $z \sim 3.4$ and 3.9 are caused by a lack of lower- $N_{\mathrm{H} \text { I }}$ and lower- $b$ lines at lower- $z$ parts of each QSO at $z>3$, probably due to the He II reionization. This result suggests that an impact from the He II reionization on the forest might be mainly on the lower- $N_{\mathrm{H} \text { I }}$ forest. From this new dataset, we find some forest clouds with a high ratio of Si IV column density to C IV column density, $N_{\mathrm{Si} \text { IV }} / N_{\mathrm{C} \text { IV }}$, at $z<2.5$, although the bulk of the forest clouds shows lower $N_{\mathrm{Si} \text { IV }} / N_{\mathrm{C} \text { IV }}$. This high $N_{\mathrm{Si} \text { IV }} / N_{\mathrm{C} \text { IV }}$ at $z<2.5$ suggests that some forest clouds are exposed to a soft UV background. This lack of strong discontinuity of $N_{\mathrm{Si} \text { IV }} / N_{\mathrm{C} \text { IV }}$ at $N_{\mathrm{H} \text { I }}=10^{14-17} \mathrm{~cm}^{-2}$ at $z \sim 3$ suggests that $N_{\mathrm{Si} \text { IV }} / N_{\mathrm{C} \text { IV }}$ might not be a good observational tool to probe the He II reionization and/or that the UV background might be strongly affected by local, high- $z$ galaxies at $z<3$.
\end{abstract}

Key words. cosmology: observations - quasars: general - intergalactic medium

\section{Introduction}

The Ly $\alpha$ forest imprinted in the spectra of high- $z$ QSOs arises from the fluctuating low-density intergalactic medium (IGM), highly photoionized by the metagalactic UV background. Since the universe expands adiabatically and the Ly $\alpha$ forest is in photoionization equilibrium with the UV background, the temperature of the Ly $\alpha$ forest as a function of $z$ provides a unique and powerful tool to probe the physical state of the IGM and the reionization history of the universe (Hui \& Gnedin 1997; Schaye et al. 1999; Ricotti, Gnedin \& Shull 2000; McDonald et al. 2000).

Send offprint requests to: T.-S. Kim, e-mail: tkim@eso.org

* The data used in this study are based on public data released from the VLT/UVES Commissioning and Science Verification and from the OPC program 65.O-0296A (P.I. S. D'Odorico) at the VLT/Kueyen telescope, ESO, Paranal, Chile.
For a low-density (the baryon overdensity $\delta<\sim 10$ ), photoionized gas, the temperature of the gas is shown to be tightly correlated with the overdensity of the gas. This relation, i.e. the equation of state, is defined by $T=T_{0}(1+$ $\delta)^{\gamma-1}$, where $T$ is the gas temperature in $\mathrm{K}, T_{0}$ is the gas temperature in $\mathrm{K}$ at the mean gas density and $(\gamma-1)$ is a constant at a given redshift $z$. Both $T_{0}$ and $(\gamma-1)$ are a function of $z$, depending on the thermal history of the IGM (Hui \& Gnedin 1997).

This equation of state, however, is not directly observable. Instead of $T$ and $(1+\delta)$, observations only provide the neutral hydrogen column density $N_{\mathrm{H} \text { I }}\left(\right.$ in $\left.\mathrm{cm}^{-2}\right)$ and the Doppler parameter $b$ (in $\mathrm{km} \mathrm{s}^{-1}$ ) of the forest absorption lines. In practice, a lower cutoff envelope in the $N_{\mathrm{H}_{\mathrm{I}}}-b$ distribution is used to probe the upper limit on the temperature of the IGM since the forest lines could be broadened by processes other than the thermal broadening. Translating a $N_{\mathrm{HI}}-b$ envelope into a $(1+\delta)-T$ 
relation depends on many physical assumptions, such as the ionizing UV background $J_{\nu}$ (Miralda-Escudé et al. 1996; Schaye et al. 1999).

This minimum Doppler cutoff $b_{\mathrm{c}}\left(N_{\mathrm{HI}}\right)$ can be described by

$\log \left(b_{\mathrm{c}}\right)=\log \left(b_{0}\right)+(\Gamma-1) \log \left(N_{\mathrm{H}}\right)$,

where $\log \left(b_{0}\right)$ is the intercept of the cutoff in the $\log \left(N_{\mathrm{H}}\right)_{-}$ $\log b$ diagram and $(\Gamma-1)$ is the slope of the cutoff (Schaye et al. 1999).

From observations alone, both no $z$-evolution of $N_{\mathrm{H} \mathrm{I}^{-}}$ independent $b_{\mathrm{c}}$ (Kirkman \& Tytler 1997; Savaglio et al. 1999) and increasing $b_{\mathrm{c}}$ with decreasing $z$ (Kim et al. 1997) have been claimed. Results from simulations combined with observations have also claimed both no- $z$ evolution of $T_{0}$ and $(\gamma-1)$ (McDonald et al. 2000) and a $z$-evolution (Ricotti et al. 2000; Schaye et al. 2000; Kim et al. 2001a). Deriving $b_{\mathrm{c}}\left(N_{\mathrm{H}}\right)$ from observations depends on many factors such as the method of line deblending, the number of available absorption lines, the metal-line contamination, and the method of fitting the lower $N_{\mathrm{HI}}-b$ envelope $(\mathrm{Hu}$ et al. 1995; Kirkman \& Tytler 1997; Bryan \& Machacek 2000; McDonald et al. 2000; Ricotti et al. 2000; Shaye et al. 2000; Kim et al. 2001a). The different approaches and the limited numbers of lines have led, in part, to the contradicting results on the evolution of $b_{\mathrm{c}}\left(N_{\mathrm{H}}\right)$ in the literature.

Here, using a new, increased dataset from 7 QSOs observed with the VLT/UVES combined with the published data on one QSO obtained with Keck/HIRES, we present the evolution of the Doppler cutoff $b_{\mathrm{c}}\left(N_{\mathrm{HI}}\right)$ at three redshifts $\langle z\rangle=2.1,3.3$ and 3.8. In particular, five QSOs at $\langle z\rangle=2.1$ enable us to study the cosmic variance of $b_{\mathrm{C}}\left(N_{\mathrm{H}}\right)$ and to improve a determination of $b_{\mathrm{C}}\left(N_{\mathrm{HI}}\right)$ at lower $z$ for the first time. In Sect. 2, we briefly describe the data used in this study. The analyses of the observations are presented in Sect. 3. The discussion is in Sect. 4 and the conclusions are summarized in Sect. 5. In this study, all the quoted uncertainties are $1 \sigma$ errors.

\section{Data used in the study}

Table 1 lists the eight QSOs analyzed in this paper. Seven QSOs were observed with the UVES spectrograph at the VLT Kueyen telescope (built by ESO, P.I. S. D'Odorico). The UVES data were reduced with the MIDAS ECHELLE/UVES package. The final reduced vacuum heliocentric spectra have $S / N$ of 30 50 per pixel in the regions of interest and a resolution of $R \sim 45000$. The spectra were normalized locally using a 5 th order polynomial fit. The normalized spectra were then fitted with Voigt profiles using VPFIT (Carswell et al.: http://www.ast.cam.ac.uk/ rfc/vpfit.html) with the reduced $\chi^{2}$ threshold of 1.3 to obtain the three line parameters, $z, b$ and $N_{\mathrm{H} \text { I }}$. The metal lines were identified and removed as described in Kim et al. (2001a). Details of the observations and data reduction, and the fitted line lists may be found in Kim et al. (2001a, 2001b).
The line parameters of Q0000-263 were taken from Lu et al. (1996) to include the highest redshift Ly $\alpha$ forest available in the literature, with similar resolution and $S / N$ to the UVES data. Their analysis of Q0000-263 was also undertaken with VPFIT.

In order to avoid confusion with the $\operatorname{Ly} \beta$ forest and the proximity effect, we consider only the wavelength range from the $\mathrm{Ly} \beta$ emission to $3000 \mathrm{~km} \mathrm{~s}^{-1}$ shortward of the Ly $\alpha$ emission. However, the redshift intervals actually used are further limited by other factors such as the incomplete coverage of the forest region, a damped Ly $\alpha$ system and our attempt to overlap the wavelengths of each QSO as much as possible to study the cosmic variance of $b_{\mathrm{c}}\left(N_{\mathrm{H}}\right)$. Table 1 lists the wavelength ranges used for each QSO.

We restrict our analysis to $N_{\mathrm{H} \text { I }}=10^{12.5-14.5} \mathrm{~cm}^{-2}$. The lower limit corresponds to the detection threshold in the regions of poorest $S / N$ and the upper limit is where the $N_{\mathrm{H} \text { I }}$ estimate from fitting $\mathrm{Ly} \alpha$ alone becomes unreliable because of line saturation. Because lines in blends can also have large uncertainties, we have further restricted the analysis to include only those lines with profile fitting errors less than $25 \%$ in $N_{\mathrm{H} \text { I }}$ and $b$ to better define the lower cutoff envelopes (Schaye et al. 2000; Kim et al. 2001a).

In this study, Sample A defines all the lines available from all QSOs which have treated as a single dataset at each $z$. We also define Sample B in order to study a fluctuation of the Doppler cutoff at similar redshifts. The spectral coverage for each QSO from the same $z$ bin is not uniform. For those QSOs with more than $\sim 600 \AA$ coverage (HE1122-1648, HE2217-2818, Q0055-269 and Q0000263), the line lists have been divided into two subsets: a group at higher redshifts and a group at lower redshifts. The rest of the QSOs do not have enough coverage to make this splitting possible and provide only one group each. We label the ensemble of these groups Sample B. Each group of Sample B spans $300 \AA-350 \AA$ and is defined to have roughly a similar redshift coverage.

\section{The physical state of the IGM}

\subsection{The z-evolution of the Doppler cutoff}

To derive $b_{\mathrm{c}}\left(N_{\mathrm{H}}\right)$, we adopted two methods: the iterative power-law fit (Schaye et al. 1999, 2000) and the smoothed $b$ power-law fit (Bryan \& Machacek 2000). From these two power-law fit methods, we derived $\log \left(b_{0, k}\right)$ and $(\Gamma-1)_{\mathrm{k}}$ of Eq. (1) for $k=\mathrm{i}$ or $\mathrm{s}$, where subscripts "i" and "s" indicate the iterative power-law fit and the smoothed $b$ power-law fit, respectively. For the iterative power-law fit, 100 bootstrap realizations were averaged with an exclusion threshold of 0.5 absolute mean deviation $(\mathrm{AMD})^{1}$. For the smoothed $b$ power-law fit, a smoothing constant of $3 \mathrm{~km} \mathrm{~s}^{-1}$ was used for each subsample having 30 lines and the robust power-law fit was applied.

\footnotetext{
${ }^{1}$ Schaye et al. (2000) adopted a threshold of 1 AMD, which results in a power law fit with higher $\chi^{2}$.
} 
Table 1. Analyzed QSOs.

\begin{tabular}{|c|c|c|c|c|c|c|}
\hline QSO & $z_{\mathrm{em}}^{\mathrm{a}}$ & $\mathrm{mag}^{\mathrm{a}}$ & $\lambda \lambda(\AA)$ & $z_{\mathrm{Ly} \alpha}$ & $\#$ of lines $^{\mathrm{b}}$ & Comments \\
\hline Q1101-264 & 2.145 & 16.0 & $3500-3778$ & $1.88-2.11$ & 69 & UVES SV, a damped system at $z=1.8386$ \\
\hline $\mathrm{J} 2233-606$ & 2.238 & 17.5 & $3500-3890$ & $1.88-2.20$ & 88 & UVES Commissioning I \\
\hline HE1122-1648 & 2.400 & 17.7 & $3500-4091$ & $1.88-2.37$ & 179 & UVES SV, split into $2^{\mathrm{c}}$ \\
\hline HE2217-2818 & 2.413 & 16.0 & $3510-4100$ & $1.89-2.37$ & 159 & UVES Commissioning I, split into $2^{\mathrm{d}}$ \\
\hline HE1347-2457 & 2.534 & 16.8 & $3760-4100$ & $2.09-2.37$ & 91 & UVES SV, incomplete observations \\
\hline Q0302-003 & 3.281 & 18.4 & $4808-5150$ & $2.96-3.24$ & 107 & UVES Commissioning I, incomplete observations \\
\hline Q0055-269 & 3.655 & 17.9 & $4850-5598$ & $2.99-3.60$ & 264 & UVES, Sep. $20-22,2000$, split into $2^{\mathrm{e}}$ \\
\hline Q0000-263 & 4.127 & 17.9 & $5450-6100$ & $3.48-4.02$ & 209 & Lu et al. (1996), split into $2^{\mathrm{f}}$ \\
\hline
\end{tabular}

a Taken from the SIMBAD database. The magnitude of HE1347-2457 is from NED.

b For $N_{\mathrm{H} \text { I }}=10^{12.5-14.5} \mathrm{~cm}^{-2}$. Only for lines with the errors less than $25 \%$ in both $N_{\mathrm{H}_{\mathrm{I}}}$ and $b$.

c For Sample B, the spectrum is split into 3500-3800 $\AA$ (87 lines) and 3800-4091 $\AA$ (92 lines).

d For Sample B, the spectrum is split into 3510-3800 $\AA$ (76 lines) and 3800-4100 $\AA$ (83 lines).

e For Sample B, the spectrum is split into 4850-5220 $\AA$ (110 lines) and 5220-5598 $\AA$ (154 lines).

f For Sample B, the spectrum is split into 5450-5820 $\AA$ (106 lines) and 5820-6100 $\AA$ (103 lines).

Table 2. The power-law fits to $b_{\mathrm{c}}\left(N_{\mathrm{H}}\right)$.

\begin{tabular}{|c|c|c|c|c|c|c|c|c|}
\hline \multicolumn{9}{|c|}{ Sample A } \\
\hline$<z>$ & $\log N_{\mathrm{H}}$ & \# of lines & $\log \left(b_{0, \mathrm{i}}\right)$ & $(\Gamma-1)_{\mathrm{i}}$ & $b_{\mathrm{c}, \mathrm{i},(13.6)}$ & $\log \left(b_{0, \mathrm{~s}}\right)$ & $(\Gamma-1)_{\mathrm{s}}$ & $b_{\mathrm{c}, \mathrm{s}}(13.6)$ \\
\hline 2.1 & $13.0-14.5$ & 349 & $-0.745 \pm 0.089$ & $0.150 \pm 0.006$ & $19.8 \pm 0.8$ & $-0.495 \pm 0.074$ & $0.131 \pm 0.002$ & $19.1 \pm 1.1$ \\
\hline 3.3 & $13.0-14.5$ & 275 & $-0.413 \pm 0.116$ & $0.122 \pm 0.008$ & $17.2 \pm 1.0$ & $0.279 \pm 0.473$ & $0.072 \pm 0.127$ & $18.4 \pm 1.5$ \\
\hline 3.8 & $13.3-14.5$ & 152 & $-0.948 \pm 0.125$ & $0.159 \pm 0.008$ & $16.8 \pm 1.6$ & $-2.699 \pm 0.159$ & $0.285 \pm 0.042$ & $14.9 \pm 1.1$ \\
\hline $2.0^{\mathrm{a}}$ & $13.0-14.5$ & 176 & $-0.214 \pm 0.180$ & $0.111 \pm 0.013$ & $19.1 \pm 1.1$ & $-1.078 \pm 0.173$ & $0.172 \pm 0.047$ & $18.0 \pm 1.1$ \\
\hline $2.2^{\mathrm{a}}$ & $13.0-14.5$ & 173 & $-0.954 \pm 0.132$ & $0.167 \pm 0.010$ & $19.7 \pm 1.0$ & $0.400 \pm 0.110$ & $0.066 \pm 0.030$ & $19.9 \pm 1.1$ \\
\hline $3.1^{\mathrm{b}}$ & $13.0-14.5$ & 157 & $0.645 \pm 0.379$ & $0.048 \pm 0.027$ & $19.9 \pm 1.5$ & $-0.366 \pm 0.103$ & $0.122 \pm 0.028$ & $19.6 \pm 1.1$ \\
\hline $3.4^{\mathrm{b}}$ & $13.0-14.5$ & 118 & $-0.330 \pm 0.053$ & $0.117 \pm 0.009$ & $17.5 \pm 0.8$ & $-0.745 \pm 0.131$ & $0.142 \pm 0.035$ & $15.5 \pm 1.1$ \\
\hline $3.6^{\mathrm{c}}$ & $13.3-14.5$ & 74 & $0.572 \pm 0.331$ & $0.054 \pm 0.023$ & $20.4 \pm 0.8$ & $1.092 \pm 0.007$ & $0.014 \pm 0.002$ & $19.0 \pm 1.0$ \\
\hline $3.9^{\mathrm{c}}$ & $13.3-14.5$ & 78 & $-0.697 \pm 0.133$ & $0.143 \pm 0.010$ & $17.5 \pm 0.7$ & $-0.499 \pm 0.235$ & $0.122 \pm 0.062$ & $14.5 \pm 1.1$ \\
\hline \multicolumn{9}{|c|}{ Sample B (averaged for the individual members) } \\
\hline 2.1 & $13.0-14.5$ & $\ldots$ & $-0.044 \pm 0.506$ & $0.102 \pm 0.037$ & $21.4 \pm 0.8$ & $0.053 \pm 0.731$ & $0.090 \pm 0.053$ & $19.2 \pm 1.1$ \\
\hline 3.3 & $13.0-14.5$ & $\ldots$ & $0.617 \pm 0.966$ & $0.051 \pm 0.067$ & $20.3 \pm 2.7$ & $0.283 \pm 0.970$ & $0.072 \pm 0.068$ & $18.3 \pm 2.7$ \\
\hline 3.8 & $13.3-14.5$ & $\ldots$ & $-0.063 \pm 0.898$ & $0.098 \pm 0.062$ & $19.0 \pm 2.1$ & $0.182 \pm 1.350$ & $0.078 \pm 0.094$ & $17.4 \pm 3.1$ \\
\hline \multicolumn{9}{|c|}{ Results from Schaye et al. (2000) (their sample corresponds to our Sample B) } \\
\hline$\sim 3.1$ & $12.5-14.5$ & $\ldots$ & $\ldots$ & $\leq 0.150$ & $\sim 23-24$ & $\ldots$ & $\ldots$ & $\ldots$ \\
\hline$\sim 3.1$ & $12.5-14.5$ & $\ldots$ & $\ldots$ & $\sim 0$ & $\sim 20-22$ & $\ldots$ & $\ldots$ & $\ldots$ \\
\hline$\sim 3.8$ & $12.5-14.8$ & $\ldots$ & $\ldots$ & $\leq 0.150$ & $\sim 18-19$ & $\ldots$ & $\ldots$ & $\ldots$ \\
\hline
\end{tabular}

${ }^{a}$ In order to have a similar number of lines at $z \sim 2.0$ and $z \sim 2.2$, the $\langle z\rangle=2$ sample consists of the line parameters from Q0011-264, J2233-606, HE1122-1648 at 3500-3760 $\AA$ and HE2217-2818 at 3510-3760 $\AA$. The the $<z>=2.2$ sample consists of the line parameters from Q1347-2457, HE1122-1648 at 3760-4100 A and HE2217-2818 at 3760-4100 A.

b The $\langle z\rangle=3.1$ sample and the $\langle z\rangle=3.4$ sample are taken from Q0302-003 and Q0055-269 at 4850-5220 $\AA$, from Q0055-269 at 5220-5598 A, respectively.

c The $\langle z\rangle=3.6$ sample and the $\langle z\rangle=3.9$ sample are taken from 5450-5820 $\AA$ and from 5820-6100 A, respectively.

Schaye et al. (2000) used the iterative power-law fit for $N_{\mathrm{H}}=10^{12.5-14.5} \mathrm{~cm}^{-2}$ at $2<z<3.7$ and for $N_{\mathrm{H} \text { I }}=10^{12.5-14.8} \mathrm{~cm}^{-2}$ at $3.7<z<4.4$. Lines with $N_{\mathrm{H} \text { I }} \leq 10^{13} \mathrm{~cm}^{-2}$, however, suffers from incompleteness due to line blending. For example, at $z \sim 2.1$, lines with $N_{\mathrm{H}} \leq 10^{13} \mathrm{~cm}^{-2}$ are affected by incompleteness and this threshold increases with $z$ (cf. Hu et al. 1995; Kim et al. 1997, 2001a, 2001b). Incompleteness causes a bias in the measurements of $(\Gamma-1)$. Due to the lack of lines with $N_{\mathrm{H} \text { I }}$ below this threshold and $b \leq 15 \mathrm{~km} \mathrm{~s}^{-1}$ (cf. Kim et al. 2001a), an observationally measured $(\Gamma-1)$ value becomes flatter than its true value. We have defined different lower $N_{\mathrm{H} \text { I }}$ fitting thresholds at the various redshifts in order to avoid this bias (see Fig. 1) and to obtain a stable estimate of $(\Gamma-1)$ and $b_{\mathrm{c}}$ at the fixed column density $N_{\mathrm{H}}=10^{13.6} \mathrm{~cm}^{-2}, b_{\mathrm{c}}(13.6)$. 


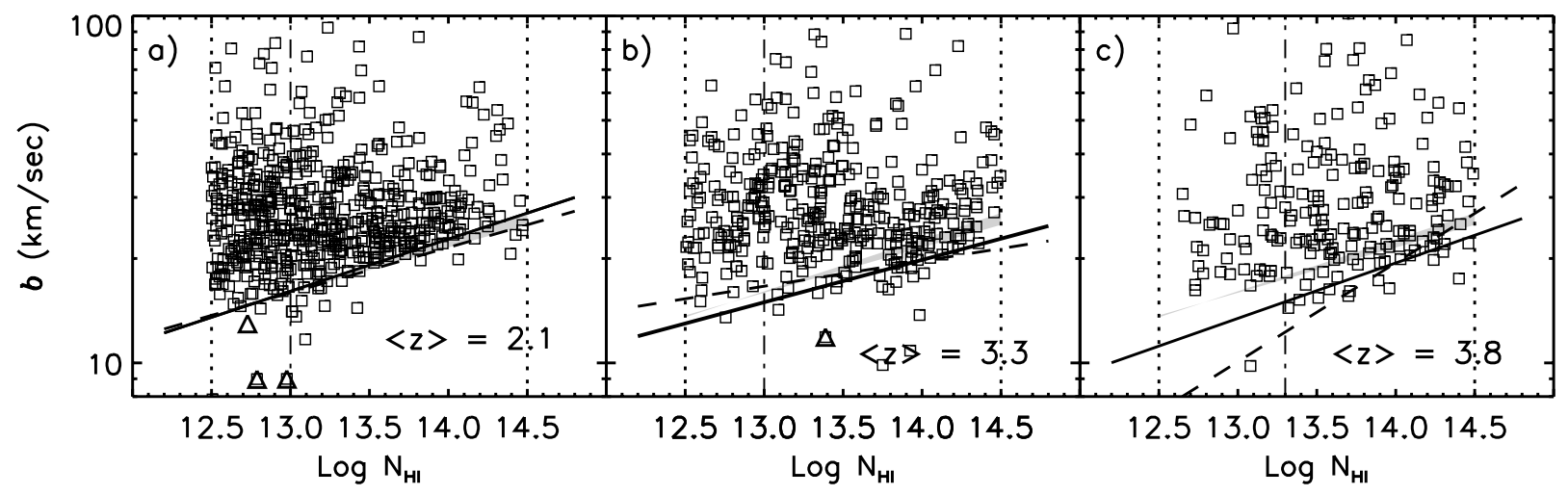

Fig. 1. The $N_{\mathrm{H}_{\mathrm{I}}}-b$ diagrams at $\langle z\rangle=2.1,3.3$ and 3.8. Errors are not displayed. Triangles indicate possible metal lines or lines in the $\mathrm{H}_{\mathrm{I}}$ complex mixed with metal lines. Dotted lines indicate the $N_{\mathrm{H}}$ ranges considered in the study. Dot-dashed lines represent the lower $N_{\mathrm{H}}$ fitting threshold actually used in the fit, above which incompleteness is negligible. Solid lines and dashed lines represent the iterative power-law fits and the smoothed $b$ power-law fits, respectively. Shaded area represents a $N_{\mathrm{H}}-b$ distribution enclosed by two power-law fits at $\langle z\rangle=2.1$.

Figure 1 shows the $N_{\mathrm{HI}}-b$ diagram at $\langle z\rangle=2.1,3.3$ and 3.8 for Sample A. The fitted parameters are listed in Table 2, as well as the $N_{\mathrm{H} \text { I }}$ ranges used in the fit. At higher $z$, several lines show a $b$ value smaller than $b_{\mathrm{c}}\left(N_{\mathrm{H}}\right)$ at $\langle z\rangle=2.1$ (shaded area). In fact, $b_{\mathrm{c}}(13.6)$ from the both power-law fits increases as $z$ decreases. The slopes $(\Gamma-1)_{\mathrm{i}}$ at $\langle z\rangle=2.1$ and 3.8 are similar within errors, while the slope $(\Gamma-1)_{\mathrm{s}}$ at $\langle z\rangle=2.1$ is flatter than the one at $\langle z\rangle=3.8$ more than $3 \sigma$. Both slopes shows the lowest value at $\langle z\rangle=3.3$ (see Table 2). Note that, however, the errors from the bootstrap method are likely to be underestimated (cf. Schaye et al. 2000). The close examination of Fig. 1 suggests that $(\Gamma-1)_{\mathrm{s}}$ at $\langle z\rangle=3.8$ might well be overestimated and that the real $(\Gamma-1)$ might be inbetween $(\Gamma-1)_{\mathrm{i}}$ and $(\Gamma-1)_{\mathrm{s}}$.

We visually compared our $b_{\mathrm{c}, \mathrm{i}}(13.6)$ with the results of Schaye et al. (2000; their Fig. 1), although their sample corresponds to our Sample B. As shown in Table 2, their $b_{\mathrm{c}}(13.6)$ values are larger than our $b_{\mathrm{c}}(13.6)$ values at similar $z$ with the differences larger at smaller $z$. Their $(\Gamma-1)_{\mathrm{i}}$ values appear to be flatter than our values at all $z$, likely caused by their lack of accounting for incompleteness of observed lines.

\subsection{Fluctuations of the Doppler cutoff}

Figure 2 shows $(\Gamma-1)_{\mathrm{i}}$ and $b_{\mathrm{c}, \mathrm{i}}(13.6)$ as a function of the lower $N_{\mathrm{H} \text { I }}$ threshold $N_{\mathrm{H} \text { I,th }}$ for Sample A. The lack of lines due to incompleteness results in flatter $(\Gamma-1)_{\mathrm{i}}$ at lower $N_{\mathrm{H} \text { I,th }}$. When the lower $N_{\mathrm{HI}}$ threshold increases so that incompleteness does not affect the $N_{\mathrm{H}_{\mathrm{I}}}-b$ distribution severely, $(\Gamma-1)_{\mathrm{i}}$ becomes stabilized (the lower $N_{\mathrm{H} \text { I,th }} \sim$ $10^{13} \mathrm{~cm}^{-2}$ at $\langle z\rangle=2.1$ and 3.3 , and the lower $N_{\mathrm{HI}, \mathrm{th}} \sim$ $10^{13.3} \mathrm{~cm}^{-2}$ at $\langle z\rangle=3.8$ ). If the available lines in the fit, however, become too small for higher $N_{\mathrm{H} \text { I,th }},(\Gamma-1)_{\mathrm{i}}$ becomes rather ill-defined. On the other hand, the lower $N_{\mathrm{H} \text { I,th }}$ does not affect the $b_{\mathrm{c}, \mathrm{i}}(13.6)$ values since $b_{\mathrm{c}, \mathrm{i}}$ at $N_{\mathrm{H}} \sim 10^{13.6} \mathrm{~cm}^{-2}$ behaves more like a pivotal point. The

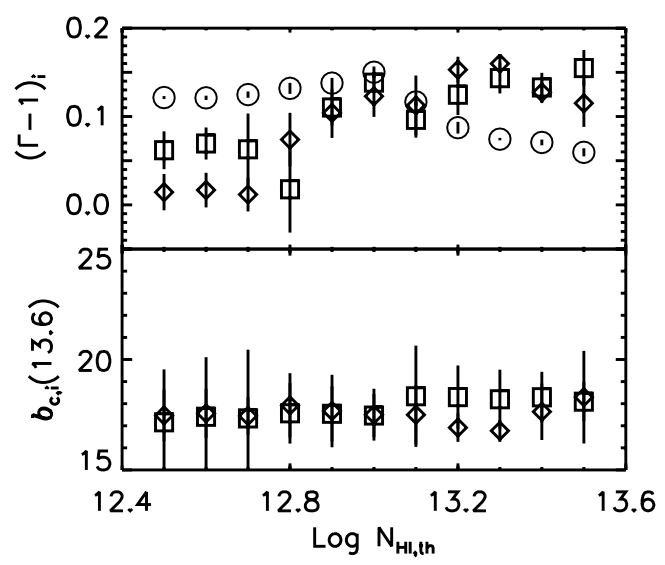

Fig. 2. Slopes $(\Gamma-1)_{\mathrm{i}}$ and $b_{\mathrm{c}, \mathrm{i}}(13.6)$ as a function of a lower $N_{\mathrm{H} \text { I }}$ threshold $N_{\mathrm{H} \text { I,th }}$ for Sample A. Open circles, open squares and open diamonds indicate $\langle z\rangle=2.1,3.3$ and 3.8 , respectively.

slopes $(\Gamma-1)_{\mathrm{s}}$ and $b_{\mathrm{c}, \mathrm{s}}(13.6)$ from the smoothed $b$ powerlaw fit also show similar behaviors.

Figure 3 shows $(\Gamma-1)_{\mathrm{i}}$ and $b_{\mathrm{c}, \mathrm{i}}(13.6)$ as a function of $z$ for the individual members of Sample B (open symbols), the averaged values from Sample B (filled triangles) and Sample A (filled symbols). The members of Sample B show the large fluctuation of $(\Gamma-1)_{\mathrm{i}}$ and $b_{\mathrm{c}, \mathrm{i}}(13.6)$ even at the similar $z$ (see Table 2). With more lines available to sharpen the lower cutoff envelope, $(\Gamma-1)_{\mathrm{i}}$ becomes steeper than the ones derived from the individual members using a smaller number of lines, possibly approaching asymptotic values (Sample A: filled circles, filled squares and filled diamonds). For both Sample A and Sample B averaged (filled triangles), $(\Gamma-1)_{\mathrm{i}}$ shows the lowest value at $\langle z\rangle=3.3$, although uncertainties for Sample B averaged are rather large at $\langle z\rangle=3.3$ and 3.8. While the $1 \sigma$ is 0.037 at $<$ $z\rangle=2.1$, it becomes twice as large as that at $\langle z\rangle=3.3$ and 3.8. This larger fluctuation at $z>3$ is caused in part by a smaller data size and in part by the cosmic variance. The fluctuation at $\langle z\rangle=2.1$ is mostly caused by different sightlines. A single, long 


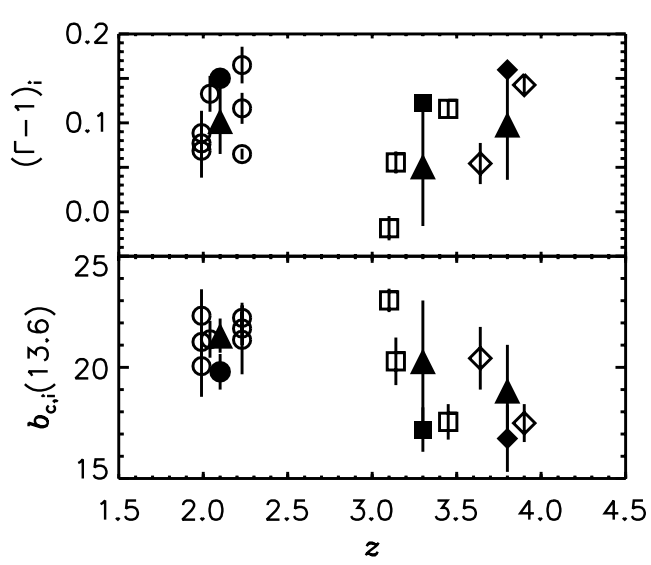

Fig. 3. Slopes $(\Gamma-1)_{\mathrm{i}}$ and $b_{\mathrm{c}, \mathrm{i}}(13.6)$ as a function of $z$. Open symbols (circles, squares and diamonds at $\langle z\rangle=2.1,3.3$ and 3.8 , respectively), filled symbols (same as in open symbols) and filled triangles represent the individual members of Sample B, Sample A and Sample B averaged at each $z$, respectively.

sightline does not show any significant difference between the lower- $z$ part and the higher- $z$ part, such as HE11221648 and HE2217-2818. On the other hand, at $\langle z\rangle=3.3$ and 3.8, even a single, long sightline shows a difference between the lower- $z$ part and the higher- $z$ part more than $3 \sigma$ (see Sect. 3.3 for more discussions).

Similarly, $b_{c, i}(13.6)$ derived from a larger number of lines is smaller, possibly approaching asymptotic values (Sample A: filled circles, filled squares and filled diamonds). Both the $b_{c, i}(13.6)$ values from Sample A and Sample B averaged (filled triangles) increase as $z$ decreases, although the uncertainties for Sample B averaged are rather large. Note again that the larger fluctuation of $b_{\mathrm{c}, \mathrm{i}}(13.6)$ at $\langle z\rangle=3.3$ and 3.8 (keep in mind the smaller sample sizes at $z>3$ at the same time). The smoothed $b$ power-law fit also produces the large fluctuations of $(\Gamma-1)_{\mathrm{s}}$ and $b_{\mathrm{c}, \mathrm{s}}(13.6)$ for the individual members of Sample B (see Table 2).

Our $(\Gamma-1)_{\mathrm{i}}$ and $b_{\mathrm{c}, \mathrm{i}}(13.6)$ for Sample A are not consistent with the Schaye et al. values by more than $3 \sigma$. Our $(\Gamma-1)_{\mathrm{i}}$ and $b_{\mathrm{c}, \mathrm{i}}(13.6)$ for Sample B, however, are completely in agreement with theirs. This result shows that deriving $(\Gamma-1)_{\mathrm{i}}$ and $b_{\mathrm{c}, \mathrm{i}}(13.6)$ depends largely on the number of lines in the fit and is subject to the cosmic variance. Experiments with the fitted line parameters show that in general more than 200 lines in the fit stabilizes the results (cf. Schaye et al. 1999). The large fluctuation found from sightline to sightline in similar redshift ranges using the number of lines smaller than 200 could explain in part the previous contradictory conclusions on the evolution of $(\Gamma-1)$ and $b_{c}(13.6)$, which were usually based on a small number of lines and of sightlines.

There are several known voids (or regions devoid of absorption lines with $N_{\mathrm{H}} \geq 10^{13.5} \mathrm{~cm}^{-2}$ ) in the present data. One void $(z \sim 3.2)$ towards Q0302-003, two $(z \sim 3.1$ and 3.3) towards Q0055-269 and three $(z \sim 1.9,2.2$ and $2.3)$ towards HE2217-2818 have been identified. The regions containing voids of Q0302-003 and Q0055-269 show a flatter $(\Gamma-1)_{\text {i }}$ by more than $7 \sigma$ (two lower squares at $z \sim 3.1$ in the upper panel of Fig. 3) and a higher $b_{\mathrm{c}, \mathrm{i}}(13.6)$ by more than $3 \sigma$ (two higher squares at $z \sim 3.1$ in the lower panel of Fig. 3) compared to the ones derived from Q0055-269 without the voids. The forest of HE2217-2818, however, does not show any significant difference between the regions with the voids and without the voids. Similarly, the HE1122-1648 forest without any recognizable voids does not show any significant difference between the lower- $z$ part and the higher- $z$ part of the spectrum.

Even though voids are produced by the enhanced radiations from local sources, the increase of the H I photoionizing photons in voids does not increase significantly the temperature of the forest as whole (Haehnelt \& Steinmetz 1998). A fixed $N_{\mathrm{H}}$, however, corresponds to a higher overdensity due to the increased photoionization. From the equation of state, a higher overdensity means a higher temperature. Therefore, if voids are produced by enhanced ionizations, there should be an increase of $b_{\mathrm{c}, \mathrm{i}}(13.6)$ and a decrease of $(\Gamma-1)_{\text {i }}$ compared to the forest without any extra heating sources at similar $z$ (Haehnelt \& Steinmetz 1998; Schaye et al. 1999).

The lack of a significant difference in $(\Gamma-1)_{\mathrm{i}}$ and $b_{\mathrm{c}, \mathrm{i}}(13.6)$ from the HE2217-2818 forest at $z \sim 2.1$ suggests that the origin of the voids towards HE2217-2818 is different. This could be due to a density fluctuation (Heap et al. 2000) and/or due to a shock heating by galactic infalls or by galactic winds (Theuns et al. 2001a).

Simulations often show that some Ly $\alpha$ lines are broadened by shock heating (Bryan \& Machacek 2000; Theuns et al. 2001a). They also show that shock heating is not very important at $z>2$ for the lower column density forest considered here. In addition, the cutoff $b$ method is less sensitive than other methods to shock-heated gas (cf. Theuns et al. 2001b).

One of the other main candidates for the fluctuations of $(\Gamma-1)_{\mathrm{i}}$ and $b_{\mathrm{c}, \mathrm{i}}(13.6)$ is the He II reionization at $z \sim 3$ (Haehnelt \& Steinmetz 1998; Songaila 1998; Schaye et al. 1999).

\subsection{The Hell reionization}

There have been various claims on a possible He II reionization at $z \sim 3$ from observations (Reimers et al. 1997; Songaila 1998; Heap et al. 2000; Kriss et al. 2001). One of the consequences of the He II reionization is an increase of the temperature of the Ly $\alpha$ forest (Haehnelt \& Steinmetz 1998). In fact, various theoretical models have predicted a $(\gamma-1) \sim 0$ (thus a flatter $(\Gamma-1))$ and a higher $T_{0}$ (thus a higher $\left.b_{\mathrm{c}}(13.6)\right)$ when the He III bubbles surrounding the ionizing sources overlap and increase the radiations in the IGM (Haehnelt \& Steinmetz 1998; Ricotti et al. 2000; Schaye et al. 2000).

To study the effect on the Ly $\alpha$ forest caused by the He II reionization, we re-grouped the individual members of Sample B. For each $z$ bin, individual members 
were divided into two subgroups according to their redshift ranges, i.e. a lower- $z$ part and a higher- $z$ part. This method of grouping has been taken since the absorption lines from the same sightline might be correlated. The number of lines at each subgroup is sampled to be similar (see Table 2).

There is no significant difference in $(\Gamma-1)_{\mathrm{i}}$ and $b_{\mathrm{c}, \mathrm{i}}(13.6)$ between $z \sim 2.0$ and $z \sim 2.1$. There is, however, a significant difference by more than $\sim 3 \sigma$ between the lower- $z$ and the higher- $z$ parts at $z \sim 3.3$ and at $z \sim 3.8$. The slope $(\Gamma-1)_{\mathrm{i}}$ decreases at lower $z$ for each bin at $z>3$, while the $b_{\mathrm{c}, \mathrm{i}}(13.6)$ increases. In short, along each line of sight at $z>3,(\Gamma-1)_{\mathrm{i}}$ decreases and $b_{\mathrm{c}, \mathrm{i}}(13.6)$ increases at $z$ decreases, as expected from the He II reionization at $z \sim 3$. This trend, however, does not continue at $z<3$, i.e. the HE1122-1648 forest and the HE2217-2818 forest do not show the similar behavior. Theuns et al. (2001b) find a similar behavior of $b$ values from the wavelet analysis using the same data presented here. This is interpreted as a result of HeII reionization at $z \sim 3.3$. They do find, however, a cold region, i.e. a region with a lower average $b$ values than the adjacent regions along HE2217-2818.

Deriving the cutoff Doppler parameters using a small number of lines introduces a large scatter (Fig. 3). Therefore, a flatter $(\Gamma-1)_{\mathrm{i}}$ and a higher $b_{\mathrm{c}, \mathrm{i}}(13.6)$ shown at the lower- $z$ part of the bins at $z>3(z \sim 3.1$ and 3.6) might be an artifact of the small number of lines in the fit. More available lines in the fit, however, tends to increase $(\Gamma-1)_{\mathrm{i}}$ and to decrease $b_{\mathrm{c}, \mathrm{i}}(13.6)$ as seen in Sect. 3.2, contrary to this result.

The $N_{\mathrm{H}}-b$ distributions at $z \sim 3.1$ and $z \sim 3.6$ show a lack of lines with $N_{\mathrm{H} \text { I }} \leq 10^{13.7} \mathrm{~cm}^{-2}$ and $b \leq 20 \mathrm{~km} \mathrm{~s}^{-1}$ with respect to the ones at $z \sim 3.4$ and 3.9, while the $N_{\mathrm{H} \text { I }}-b$ distributions for higher- $N_{\mathrm{H} \text { I }}$ lines are similar (the figures not shown). This lack of lower- $N_{\mathrm{H} \text { I }}$ and lower- $b$ lines results in a flatter $(\Gamma-1)_{\mathrm{i}}$ and a higher $b_{\mathrm{c}, \mathrm{i}}(13.6)$ at $z \sim 3.1$ and 3.6. This, however, can not be caused by incompleteness due to line blending. The number of lower$N_{\mathrm{H} \text { I }}$ lines are similar at $z \sim 3.1$ and $z \sim 3.4$, and at $z \sim 3.6$ and $z \sim 3.9$, i.e. $b$ values corresponding to lower- $N_{\mathrm{H}}$ lines are higher at $z \sim 3.1$ and $z \sim 3.6$ than those at $z \sim 3.4$ and $z \sim 3.9$. In addition, line blending is more severe at higher $z$ and should have resulted in a lack of lower- $N_{\mathrm{H} \text { I }}$ and lower- $b$ lines at $z \sim 3.4$ and $z \sim 3.9$ rather than at $z \sim 3.1$ and $z \sim 3.6$ as observed. What is observed at $z \sim 3.1$ and $z \sim 3.6$ shows the opposite behavior expected from line blending. In fact, the lower- $N_{\mathrm{H} \text { I }}$ and higher- $b$ lines seen at $z \sim 3.1$ and at $z \sim 3.6$ are expected from the He II reionization since it has a greater effect on the lower- $N_{\mathrm{H} \text { I }}$ forest than on the higher- $N_{\mathrm{H} \text { I }}$ forest (Theuns 2001, private communication).

Songaila (1998) found that there is an abrupt, sharp discontinuity in the ratio of Si IV column density to C IV column density, $N_{\mathrm{Si} \text { IV }} / N_{\mathrm{C} \text { IV }}$, at $z \sim 3$, below which $N_{\text {Si IV }} / N_{\text {C IV }}$ is always less than $\sim 0.07$. The observed $N_{\text {Si IV }} / N_{\text {C IV }}$ by Songaila (1998) suggests that the UV background is softer than a QSO-dominated background at $z>3$ and becomes harder as expected from a QSOdominated background at $z<3$. This observation has been interpreted as the complete He II reionization by $z \sim 3$, i.e. the overlap of the He III bubbles.

Figure 4 shows $N_{\mathrm{Si} \text { IV }} / N_{\mathrm{C} \text { IV }}$ of the Ly $\alpha$ forest at $N_{\mathrm{H} \text { I }}=$ $10^{14-17} \mathrm{~cm}^{-2}$ as a function of $z$ from the QSOs in Table 1 except from Q0000-263 (Kim et al. 2001, in preparation). We only include the H I systems having higher Lyman lines other than $\operatorname{Ly} \alpha$, such as $\operatorname{Ly} \beta$, Ly $\gamma$, etc. This selection enables us to estimate an accurate $\mathrm{H}$ I column density and to assign Si IV and C IV to a H I line more reliably. Note that there are no data points at $2.6<z<2.9$ at which the discontinuity of $N_{\mathrm{Si} \text { IV }} / N_{\mathrm{C} \text { IV }}$ has been reported to be the largest. Although the bulk of the forest shows lower $N_{\text {Si IV }} / N_{\text {CIV }}$ at $z<2.6$, there are the forest lines with $N_{\text {Si IV }} / N_{\text {C IV }}$ larger than 0.07 . The similar results have been reported at $z>2$ by Boksenberg et al. (1998). Bear in mind that no specific H I column density range used in their Fig. 1 is given in their paper. In addition, note that their results are based on the individual, fitted components, while ours are based on the integrated absorption lines. Using the component-by-component analysis introduces an additional scatter in the diagram due to the different velocity structures in Si IV and C IV. The number of components for one integrated system in our data is in general from 1 to 3 . The component-by-component analysis on our data also shows the similar trend found in Fig. 4 from the integrated profiles.

Our observations do not suggest any abrupt change in $N_{\text {Si IV }} / N_{\text {C IV }}$ at $1.6<z<3.6$, i.e. no abrupt change in the softness parameter in general. This result suggests that some forest clouds are exposed to a soft UV background at $z<2.6$. The lack of a sharp change in $N_{\text {Si IV }} / N_{\text {CIV }}$ at $z \sim 3$ does not mean, however, that the He II reionization did not occur at $z \sim 3$. Rather, our $N_{\mathrm{Si} \text { IV }} / N_{\mathrm{C} \text { IV }}$ indicates that $N_{\mathrm{Si} \text { IV }} / N_{\mathrm{C} \text { Iv }}$ might not be a good observational tool to probe the He II reionization. What it probes is a softness parameter of the UV background. Increasing evidences of the contribution to the UV background from local, high- $z$ galaxies would explain our high $N_{\mathrm{Si} \text { IV }} / N_{\text {C IV }}$ at $z<2.6$ without any difficulty (Giroux \& Shull 1997; Shull et al. 1999; Bianchi et al. 2001; Steidel et al. 2001). In addition, the lack of strong $z$-evolution of $(\Gamma-1)$ found for $N_{\mathrm{H} \text { I }}=$ $10^{13.7-14.5} \mathrm{~cm}^{-2}$ at $z>3$ is in agreement with the lack of strong evolution of $N_{\mathrm{Si} \text { IV }} / N_{\mathrm{C} \text { IV }}$ at $N_{\mathrm{H}}=10^{14-17} \mathrm{~cm}^{-2}$. In short, the He II reionization at $z \sim 3$ (Reimers et al. 1997; Heap et al. 2000; Smette et al. 2002) shows its impact on the Ly $\alpha$ forest mainly at $N_{\mathrm{H}_{\mathrm{I}}} \leq 10^{13.7} \mathrm{~cm}^{-2}$ and might be very inhomogeneous. Its strength might not be as strong as previously suggested and $N_{\mathrm{Si} \text { IV }} / N_{\mathrm{C} \text { IV }}$ might not be a best observational tool to probe the He II reionization.

\section{Discussion}

Converting $(\Gamma-1)$ and $b_{\mathrm{c}}(13.6)$ to the corresponding $(\gamma-1)$ and $T_{0}$ depends on many uncertain parameters, such as the UV background and the reionization history. If the UV background is dominated by QSOs without 


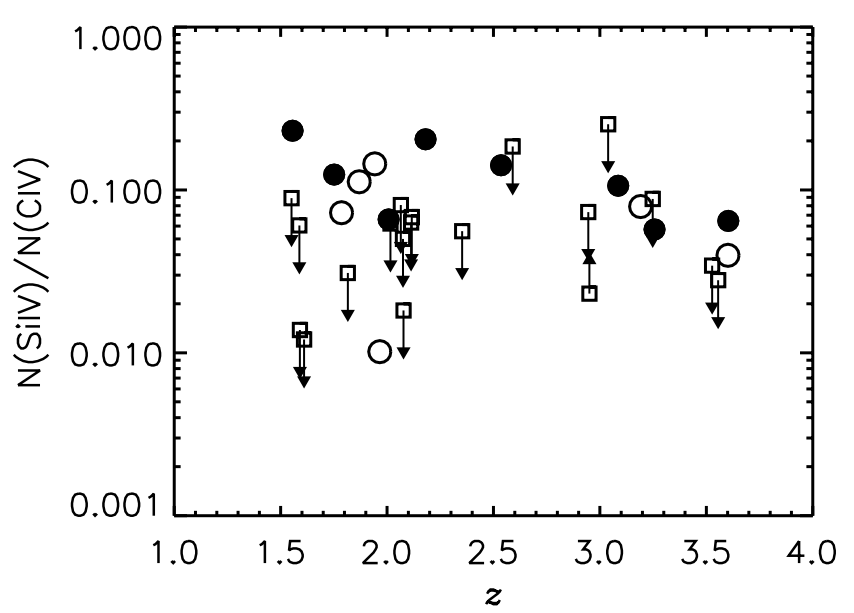

Fig. 4. The ratio of $N_{\mathrm{Si} \text { IV }} / N_{\mathrm{C} \text { IV }}$ as a function of $z$. Only the Ly $\alpha$ forest with $N_{\mathrm{H}} \leq 10^{17} \mathrm{~cm}^{-2}$ is included. Filled circles represent a forest with $N_{\text {CIV }}=10^{12.7-14} \mathrm{~cm}^{-2}$, while open circles represent a forest with $N_{\mathrm{C} \text { IV }}>10^{14} \mathrm{~cm}^{-2}$. Open squares represent upper limits or lower limits.

any extra heating, $T_{0}$ decreases and $(\gamma-1)$ increases as $z$ decreases, until they approach asymptotic values. The $b_{\mathrm{c}}(13.6)$ value, however, still increases with decreasing $z$, until it approaches an asymptotic value and finally decreases again at $z<2$ (Schaye et al. 1999).

Instead of converting to $T_{0}$, we compare our $b_{\mathrm{c}, \mathrm{i}}(13.6)$ with Figs. 3 and 4 of Schaye et al. (2000) from their simulations assuming the QSO-dominated Haardt-Madau UV background, $J_{\mathrm{HM}}$ (Haardt \& Madau 1996) ${ }^{2}$. Our $b_{\mathrm{c}}(13.6)$ is in agreement with their simulated $b_{\mathrm{c}}(13.6)$ values without extra He II heating, although the difference increases as $z$ decreases (ours being a factor of 1.1 lower at $z \sim 2$ ). It is not clear what causes their numerical simulations without the HeII reionization produce their $b_{\mathrm{c}}(13.6)$ similar to ours which suggest the extra heating. One of the explanations could be a weaker effect on the forest from the He II reionization than their simulations suggest. When $T_{0}$ is converted from the same simulations (their Fig. 2), $T_{0}$ decrease as $z$ decreases.

For $(\gamma-1)$, we assume the conversion law between $N_{\mathrm{H} \text { I }}$ and $\delta$ by Schaye (2001) assuming $J_{\mathrm{HM}}$ and $T \sim 59.2 b^{2}$ for thermally broadened lines. The conversion law is defined by

$$
\begin{aligned}
& N_{\mathrm{H} \text { I }} \sim 2.7 \times 10^{13}(1+\delta)^{1.5-0.26(\gamma-1)} T_{0,4}^{-0.26} \Gamma_{\mathrm{H} \mathrm{I}, 12}^{-1} \\
& \times\left(\frac{1+z}{4}\right)^{4.5}\left(\frac{\Omega_{\mathrm{b}} h^{2}}{0.02}\right)^{1.5}\left(\frac{f_{\mathrm{g}}}{0.16}\right)^{0.5} \mathrm{~cm}^{-2},
\end{aligned}
$$

\footnotetext{
2 The previous section suggests there is a contribution from sources other than QSOs to the UV background. Estimates of contributions from galaxies depend on many factors, such as the galaxy luminosity function and the galaxy emissivities. Including a contribution from galaxies, Shull et al. (1999) derive a UV background decreasing from $z \sim 2.5$ to $z \sim 1$, similar to the QSO-dominated $J_{\mathrm{HM}}$. On the other hand, Bianchi et al. (2001) find a non-decreasing $J$ from $z \sim 5$ to $z \sim 1$.
}

where $T_{0} \equiv T_{0,4} \times 10^{4} \mathrm{~K}$, the $\mathrm{H}$ I photoionization rate $\Gamma_{\mathrm{H} \text { I }} \equiv \Gamma_{\mathrm{H}, 12} \times 10^{12} \mathrm{~s}^{-1}, \Omega_{\mathrm{b}}$ is the baryon density, $h$ is the Hubble constant divided by 100 , and $f_{\mathrm{g}}$ is the fraction of the mass in gas (Schaye 2001). We read $T_{0}$ from Fig. 3 of Schaye et al. (2000) and $\Gamma_{\mathrm{H}}$ from Fig. 8 of Haardt \& Madau (1996), while we assume $\Omega_{\mathrm{b}} h^{2}=0.02$ and $f_{\mathrm{g}}=$ 0.16. At $\langle z\rangle=2.1,3.3$ and 3.8, $(\gamma-1) \sim 0.417,0.386$ and 0.441 . Assuming $J_{\mathrm{HM}}$, there is no clear $z$-evolution of $(\gamma-1)$ within large uncertainties. Our values and Schaye et al.'s $((\gamma-1) \sim 0.4,0.35$ and 0.25 at $\langle z\rangle=2.1,3.3$ and 3.8 assuming $J_{\mathrm{HM}}$ ) agree at $\langle z\rangle=2.1,3.3$, while our value is a factor of 1.8 larger than theirs at $\langle z\rangle=3.8$. Our $(\gamma-1)$ values agree with those of McDonald et al. at the similar $z$ ranges within uncertainties, although their simulations do not assume $J_{\mathrm{HM}}$.

\section{Conclusions}

Using the new, large dataset from high $S / N$, high resolution VLT/UVES data combined with one Keck/HIRES QSO in the literature, the minimum cutoff Doppler parameter as a function of $N_{\mathrm{H} \text { I }}, b_{\mathrm{c}}\left(N_{\mathrm{H}}\right)$, of the Ly $\alpha$ forest has been derived at $\langle z\rangle=2.1,3.3$ and 3.8. We have found:

1. When incompleteness is accounted for, the derived $(\Gamma-1)$ is consistent with no- $z$ evolution with a suggestion of lower $(\Gamma-1)$ at $z \sim 3.1$, while $b_{\mathrm{c}}(13.6)$ increases as $z$ decreases. These results suggest that $(\gamma-1)$ shows no $z$-evolution within uncertainties and that $T_{0}$ decreases as $z$ decreases, assuming the QSO-dominated UV background from Haardt \& Madau (1996).

2 . The $(\Gamma-1)$ and $b_{\mathrm{c}}(13.6)$ values show a large fluctuation when derived using a subsample even at the similar redshifts. The fluctuation is larger at $z>3$ than at $z<3$. Although smaller number of lines in the fit could result in this fluctuation, we use a similar number of lines to derive $(\Gamma-1)$ and $b_{c}(13.6)$ for each subsample. This result suggests that there might be a large fluctuation in the IGM temperature along different sightlines even at similar $z$.

3. There is a suggestion of a flatter $(\Gamma-1)$ and higher $b_{\mathrm{c}}(13.6)$ along each line of sight as $z$ decreases at $z>3$ more than $3 \sigma$, probably due to the He II reionization. At $z \sim 2.1$, there is no such a significant trend along each line of sight. This result occurs due to the lack of lower- $N_{\mathrm{H} \text { I }}$ and smaller- $b$ lines in the lower- $z$ part of the spectra at $z>3$. Our result implies that the impact of the HeII reionization on the Ly $\alpha$ forest might be mainly on the lower- $N_{\mathrm{H}}$ forest and that its significance might be smaller than previously suggested.

4. The lack of strong discontinuity of $N_{\mathrm{Si} \text { IV }} / N_{\mathrm{C} \text { IV }}$ at $N_{\mathrm{H} \text { I }}=10^{14-17} \mathrm{~cm}^{-2}$ at $z \sim 3$ suggests that $N_{\text {Si IV }} / N_{\text {CIV }}$ might not be a good observational tool to probe the He II reionization and that the UV background might be strongly contributed by local, high- $z$ galaxies at $z<3$. 
Acknowledgements. We are indebted to M. DessaugesZavadsky for support during the observations of Q0055-269. We thank Martin Haehnelt, Joop Schaye, Tom Theuns and Saleem Zaroubi for insightful discussions. TSK thanks Dave Jewitt, Bob Carswell and Glenn Morris for their careful reading of the manuscript. This work has been conducted with partial support by the Research Training Network "The Physics of the Intergalactic Medium" set up by the European Community under the contract HPRN-CT2000-00126 RG29185 and by ASI through contract ARS-98-226.

\section{References}

Bianchi, S., Cristiani, S., \& Kim, T.-S. 2001, A\&A, 376, 1

Boksenberg, A., Sargent, W. L. W., \& Rauch, M. 1998, in The birth of galaxies, ed. B. Guiderdoni, et al. (The Gioi Publishers), 429

Bryan, G. L., \& Machacek, M. E. 2000, ApJ, 534, 57

Giroux, M. L., \& Shull, J. M. 1997, AJ, 113, 1505

Haardt, F., \& Madau, P. 1996, ApJ, 461, 20

Haehnelt, M. G., \& Steinmetz, M. 1998, MNRAS, 298, 21

Heap, S. R., Williger, G. M., Smette, A., et al. 2000, ApJ, 534, 69

Hu, E. M., Kim, T.-S., Cowie, L. L., Songaila, A., \& Rauch, M. 1995, AJ, 110,

Hui, L., \& Gnedin, N. Y. 1997, MNRAS, 292, 27

Kim, T.-S., Hu, E. M., Cowie, L. L., \& Songaila, A. 1997, AJ, 114,1526

Kim, T.-S., Cristiani, S., \& D'Odorico, S. 2001a, A\&A, 373, 757
Kim, T.-S., Carswell, R. F., Cristiani, S., D'Odorico, S., \& Giallongo, E. 2001b, MNRAS, submitted

Kirkman, D., \& Tytler, D. 1997, AJ, 484, 672

Kriss, G. A., Shull, J. M., Oegerle, W., et al. 2001, Science, 293, 1112

Lu, L., Sargent, W. L. W., Womble, D. S., \& Takada-Hidai, M. 1996, ApJ, 472, 509

McDonald, P., Miralda-Escudé, J., Rauch, M., et al. 2000, ApJ, 543,1

Miralda-Escudé, J., Cen, R., Ostriker, J. P., \& Rauch, M. 1996, ApJ, 471, 582

Reimers, D., Köhler, S., Wisotzki, L., et al. 1997, A\&A, 327, 890

Ricotti, M., Gnedin, N. Y., \& Shull, J. M. 2000, ApJ, 534, 41

Savaglio, S., Ferguson, H. C., Brown, T. M., et al. 1999, ApJ, $515, \mathrm{~L} 5$

Schaye, J., Theuns, T., Leonard, A., \& Efstathiou, G. 1999, MNRAS, 310, 57

Schaye, J., Theuns, T., Rauch, M., Efstathiou, G., \& Sargent, W. L. W. 2000, MNRAS, 318, 817

Schaye, J. 2001, ApJ, 559, 507

Shull, J. M., Roberts, D., Giroux, M., Penton, S. V., \& Fardal, M. A. 1999, AJ, 118, 1450

Smette, A., Heap, S., Williger, G. M., et al. 2002, ApJ, 564, 542

Songaila, A. 1998, AJ, 115, 2184

Steidel, C. C., Pettini, M., \& Adelberger, K. L. 2001, ApJ, 546, 665

Theuns, T., Mo, H.-J., \& Schaye, J. 2001a, MNRAS, 321, 450

Theuns, T., Zaroubi, S., Kim, T.-S., Tzanavaris, P., \& Carswell, R. F. 2001b, MNRAS, submitted [astro-ph/0110600] 